\title{
Optimal Modulation Techniques of a DAB Based Isolated Bidirectional Single-stage Single-phase AC-DC Converter
}

This paper was downloaded from TechRxiv (https://www.techrxiv.org).

LICENSE

CC BY 4.0

SUBMISSION DATE / POSTED DATE

07-09-2021 / 17-09-2021

\section{CITATION}

Das, Dibakar; Basu, Kaushik; Paul, Sayan (2021): Optimal Modulation Techniques of a DAB Based Isolated Bidirectional Single-stage Single-phase AC-DC Converter. TechRxiv. Preprint.

https://doi.org/10.36227/techrxiv.16578068.v1

DOI

10.36227/techrxiv.16578068.v1 


\title{
Optimal Modulation Techniques of a DAB Based Isolated Bidirectional Single-stage Single-phase AC-DC Converter
}

\author{
Dibakar Das, Student Member, IEEE, Sayan Paul, Kaushik Basu, Senior Member, IEEE
}

\begin{abstract}
A bidirectional single-phase AC to DC converter with high-frequency isolation finds a wide range of applications, including charging electric vehicles in the vehicle to grid applications. A conventional two-stage solution, where an AC-DC front-end rectifier is connected to an isolated DC-DC converter, suffers from poor efficiency due to hard switching of the AC-DC stage and poor reliability due to the existence of an electrolytic capacitance at the interstage DC link. A Dual Active Bridge (DAB) based single-stage AC-DC converter with a potential of bidirectional power flow can overcome the problems of a twostage solution. A rich literature exists in search for finding an efficient modulation strategy for this converter. This paper presents two constant switching frequency modulation strategies that support bidirectional power flow at any power factor utilizing all three degrees of freedom in modulation, also known as triple phase-shift modulation (TPS). One of the strategies minimizes RMS high-frequency transformer current over the line cycle, and the other one optimizes peak current. Hence, the conduction loss and the component stress over the entire line cycle are minimized. ZVS conditions are met for all high-frequency switching devices for the whole operating region, while the $\mathrm{AC}$ side converter is line frequency switched, incurring negligible switching loss. AC line current waveforms are of high quality and free from low-frequency harmonics. UPF operation is of importance for single-phase power conversion. All possible modes that the converter will experience over a line cycle for UPF operation are elaborated through detailed analysis. The proposed strategies are validated through experiment and simulation with $230 \mathrm{~V}, 50 \mathrm{~Hz}$ AC grid, $400 \mathrm{~V} \mathrm{DC}$, UPF, and output power of 1.2 kW.
\end{abstract}

\section{INTRODUCTION}

Single-phase AC-DC power converters have a wide range of applications such as electric vehicle charging, energy storage systems, data center power supplies, telecommunication power supplies, DC distribution systems, etc. [1], [2]. Several applications, such as vehicle to grid operation of EV chargers, require bidirectional power flow and galvanic isolation. Conventional isolated AC-DC power conversion systems have two-stage architecture [3], [4]. An active front-end rectifier exchanges power between the $\mathrm{AC}$ grid and intermediate DCbus. An isolated DC-DC power converter is placed between the intermediate DC link and the output DC voltage for isolation and voltage matching. A resonant converter [5] or dual active bridge (DAB) [6] converter may be used for isolated DC-DC power conversion. Multiple stages of power conversion lead to reduced efficiency. AC-DC converter is hard-switched. Moreover, an electrolytic capacitor is required on the intermediate DC link, which reduces the reliability. Several single-stage power conversion topologies have been proposed in literature [7]-[9] to address these issues.

DAB-based DC-DC converter has recently gained popularity for its desirable features such as galvanic isolation, soft

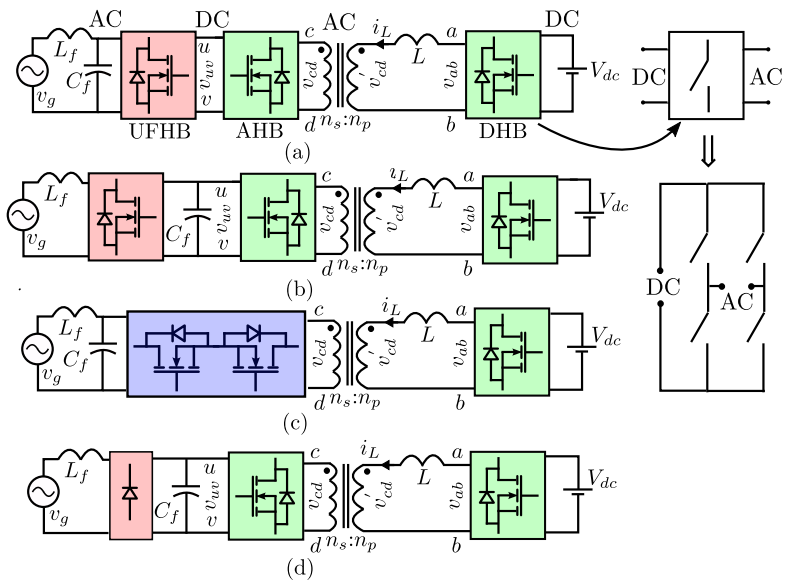

Fig. 1: Different possible configurations for single-stage DCAC power conversion

switching, bidirectional power flow capability, etc. A DCDC DAB converter uses H-bridges to convert DC voltages to high-frequency square waveforms, which are applied to the windings of a high-frequency transformer in series with an inductance. A phase shift between the square waves is introduced for power transfer. This strategy is known as singlephase-shift (SPS) in literature [6]. Later, it was found that the losses in the converter can be minimized by introducing duty modulation on the voltage waveforms, which is known as triple phase shift (TPS) in literature [10]. In this case, both the bridges apply quasi-square waveforms. Optimal TPS strategy of DC-DC DAB converter has been derived in the literature that minimizes either RMS [10], [11] or the peak [12] of the series inductor current and ensures that the soft-switching or zero-voltage-switching (ZVS) conditions are met for the given power transfer and sensed two DC port voltages.

Fig. 1 shows possible topologies of DAB-based single-stage single-phase AC-DC converter. The AC side unfolder H-bridge (UFHB) of Fig. 1a converts the line-frequency $\mathrm{AC}$ to its rectified form. The $\mathrm{AC}$ side $\mathrm{H}$-bridge $(\mathrm{AHB})$ and the $\mathrm{DC}$ side $\mathrm{H}$-bridge (DHB) form the dual bridges of the DAB. The AHB converts the rectified $\mathrm{AC}$ to a quasi-square wave $\mathrm{AC}$ whose amplitude has line-frequency sinusoidal envelop, and DHB converts DC to a quasi-square wave with fixed amplitude. So, this converter also has three degrees of modulation like the DC-DC DAB: two duty cycles of the quasi-square waves and the phase shift between each other (TPS). Input LC filter of Fig. 1a prevents the high-frequency components of the current (generated by DAB converter) from flowing into the AC grid. A slightly different topology, as shown in Fig. 1b, is obtained by moving the filter capacitor $C_{f}$ between UFHB and AHB, but the operation remains unchanged. The functionalities of 
UFHB and AHB can be combined to form an H-bridge with four-quadrant switches, as shown in Fig. 1c. Fig. 1d is the unidirectional AC-DC converter where the UFHB of Fig. 1b is replaced by a diode-bridge.

Topology of Fig. 1c is used in [13] where the AHB is square-wave modulated, and duty modulation is introduced in the DHB to improve the soft-switching range. An SPS-based strategy with the topology of Fig. 1d is discussed in [14]. [15] proposed a modulation strategy based on the topology of Fig. 1c, which supports bidirectional power flow at unity power factor (UPF) and also ensures zero-current-switching (ZCS) of AHB and ZVS of DHB. The proposed strategy, however, leads to large circulating currents and hence reduced efficiency. A variation of this strategy is proposed in [16] which results in lower inductor RMS current but at the cost of injecting low-frequency harmonic currents into the grid. A TPS-based strategy to improve soft-switching performance and efficiency at light-load operation is proposed in [17]. A genetic algorithm-based optimal TPS strategy is used to find the switching frequency at which both the inductor peak current and loss of the converter can be minimized. TPS modulation strategies, where the parameters are fixed from power and ZVS conditions, are discussed in [18] (topology Fig. 1c) and [19] (topology Fig. 1d). These strategies lead to line current distortions and low-frequency harmonics. Due to the triangular current operation, the strategy of [19] results in higher losses in the DAB converter. A TPS strategy that minimizes RMS inductor current for unidirectional power flow from AC to DC (topology Fig. 1d) is proposed in [20].

Several modulation strategies introduce switching frequency variation over line cycle along with duty modulation of the DAB voltages to improve the soft-switching performance, line current profile, and minimize RMS or peak of the inductor current [21]-[25]. A numerical optimization strategy to decide the modulation parameters is discussed in [21] (Topology: Fig.1b). Additional inductances are added across the transformer primary and secondary windings to improve the soft-switching range. This, however, increases the losses and device ratings. A hybrid strategy, consisting of phaseshift and variable frequency control, is proposed in [23]. A dual-phase-shift based strategy is used in [22], [24], [25] with numerical optimization of peak current over the line cycle (Topology: Fig.1b for [24] \& Fig.1c for [22], [25]). The strategies employing variable switching frequency lead to distortions in line-current near zero crossings for light load operation (due to clamping of switching frequency to its limit). Moreover, a converter design that needs to operate over a range of switching frequencies is complicated.

Given the above literature, it is clear that, unlike the DCDC case, the problem of finding an optimal TPS for a given active and reactive power and sensed AC and DC port voltages is not fully addressed yet. This paper presents two optimal TPS modulation strategies in section-II with the following features:

- Bi-directional power flow at any power factor, applicable to topologies Fig. 1a-c. The topology in Fig. 1d can be utilized for $\mathrm{AC}$ to $\mathrm{DC}$ power flow at UPF.

- Constant switching frequency modulation.
- One strategy minimizes inductor RMS current over the entire line cycle, while the other optimizes the maximum peak inductor current. Minimization of RMS inductor current minimizes conduction loss. The peak current minimization reduces electrical stress on the components leading to a smaller size, lower cost, and improved reliability.

- The analysis is done in time-domain for greater accuracy.

- ZVS or soft switching conditions are met over the entire line cycle for AHB and DHB while UFHB incurs negligible switching loss due to line frequency switching.

- The line current drawn from the AC grid is of high quality and free of any low-frequency harmonics.

As unity power-factor (UPF) operation is the most important case for single-phase AC-DC converter, a detailed analysis of different modes of UPF operation is provided in sectionIII. The effectiveness of the proposed modulation techniques is validated through simulation and experimental results in section-IV, and finally, the paper is concluded in section-V.

\section{THE PROPOSED OPTIMAL MODULATION STRATEGIES}

Consider a single stage DC-AC converter with DC voltage $V_{d c}$, grid voltage of peak value $V_{p k}$ and line frequency $f$. The instantaneous grid voltage $v_{g}(t)$ is given by (1), where $\omega=2 \pi f$. The topology of a single stage DC-AC power converter is shown in Fig.2. It consist of an unfolder $\mathrm{H}$ Bridge (UFHB) followed by the AC side (AHB) and DC side (DHB) H-bridge of the Dual Active Bridge (DAB) conversion stage. All the H-bridge converters have two quadrant switches as shown in Fig.2. The topology shown in Fig.2 supports bidirectional power flow at any power factor. The UFHB is switched at line frequency $f$, therefore, rectified voltage across $u$ and $v$ is given by (2).

$$
v_{g}(t)=V_{p k} \sin (\omega t) \quad \text { (1) } \quad v_{u v}(t)=V_{p k}|\sin (\omega t)|
$$

AHB and DHB form the two H-bridges of a classical DAB converter. $\mathrm{DHB}$ and $\mathrm{AHB}$ are modulated to generate quasi square waveforms $v_{a b}$ and $v_{c d}$ respectively at high frequency $f_{s}=\frac{1}{T_{s}}\left(f_{s}>>f\right)$. The duty cycles $d_{p}$ (of $\left.v_{a b}\right), d_{s}$ (of $\left.v_{c d}\right)$ and the phase shift $\delta$ between them can be adjusted to control the power transfer. This strategy is hereafter referred

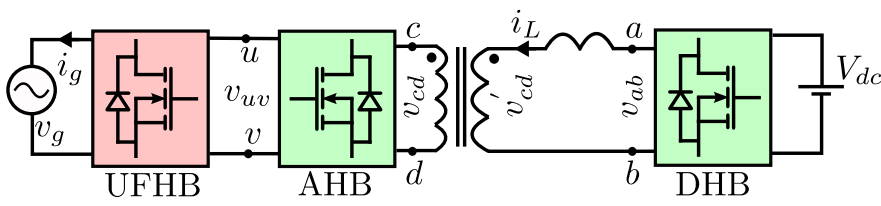

Fig. 2: Topology of the single stage DC-AC power converter
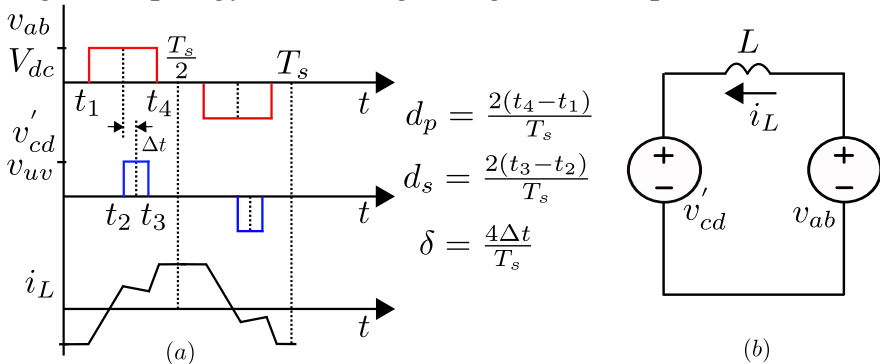

(a)

(b)

Fig. 3: (a) Typical waveforms in DAB converter with TPS modulation (b) DAB Equivalent circuit 
to as Triple phase shift (TPS) modulation. Typical waveforms of $v_{a b}, v_{c d}^{\prime}$ and $i_{L}$ with TPS modulation are shown in Fig.3a. Considering ideal operation of all the switching devices and assuming the magnetizing inductance to be large, the DAB converter can be represented by the equivalent circuit in Fig.3b.

The objective of this paper is to determine the optimal switching strategy $\left(d_{p}, d_{s}\right.$ and $\left.\delta\right)$ for the converter topology shown in Fig.1a. This strategy results in exchange of an average power $P$ (which can be bidirectional) at a power factor $\cos (\phi)$ between $V_{d c}$ and $v_{g}$. Here the power is averaged over one cycle of AC voltage. The values of $V_{p k}, V_{d c}, f$, $f_{s}$, transformer turns ratio $n\left(n=n_{p}: n_{s}\right)$ and inductance $L$ are given in the problem. The subsequent section presents the optimal switching strategy for a given set of design and operating specifications.

Consider that the converter is supplying an active power of $P$ and reactive power $Q$ to the grid whose voltage is given by (1). The average grid current over a switching cycle is given by (3), where $I_{p k}$ is the peak value of average grid current, $P=\frac{1}{2} V_{p k} I_{p k} \cos \phi$ and $Q=\frac{1}{2} V_{p k} I_{p k} \sin \phi$. For simplifying the analysis, a per-unit system is defined where $V_{b}=V_{d c}$, $Z_{b}=2 \pi f_{s} L, I_{b}=V_{b} / Z_{b}$ and $S_{b}=V_{b} I_{b}$. The average perunit power transferred in a switching cycle $\langle p\rangle_{T_{s}}(t)$ (hereafter referred as $p(t))$ is given by (4), where $\hat{p}=\frac{4 \pi f_{s} L P}{V_{d c}^{2}}$ and $\hat{s}=$ $\frac{4 \pi f_{s} L Q}{V_{d c}^{2}}$ are the peak values of per-unit active and reactive power respectively. The voltage conversion ratio is defined as (5), where $M=\frac{n V_{p k}}{V_{d c}}$.

$$
\begin{gathered}
\left\langle i_{g}\right\rangle_{T_{s}}(t)=I_{p k} \sin (\omega t-\phi) \\
\langle p\rangle_{T_{s}}(t)=\frac{v_{g}(t)\left\langle i_{g}\right\rangle_{T_{s}}(t)}{S_{b}}=[\hat{p} \sin (\omega t)-\hat{q} \cos (\omega t)] \sin (\omega t) \\
m(t)=\frac{n v_{u v}}{V_{d c}}=M|\sin (\omega t)|
\end{gathered}
$$

For a given $P$ and $Q$ and a given set of design specifications, $m(t)$ and $p(t)$ are generated which vary over a line cycle. Consider now that the unfolder voltage $v_{u v}$ is constant. This means that $m$ does not vary with time. Assuming that $p$ is also constant, the situation is similar to a DC-DC DAB operation. The inductor rms and peak currents over a switching cycle, $\left\langle i_{L r m s}\right\rangle_{T_{s}}$ and $\left.i_{L p k}\right|_{T_{s}}$, respectively, can be written as the products of base current $I_{b}$ and the per-unit currents, $i_{r m s}$ and $i_{p k}$, respectively, which are function of $D=\left[m, d_{p}, d_{s}, \delta\right]$ [26], as shown in (6) and (7).

$$
\frac{\left\langle i_{L r m s}\right\rangle_{T_{s}}}{I_{b}}=i_{r m s}(D) \quad \text { (6) } \quad \frac{\left.i_{L p k}\right|_{T_{s}}}{I_{b}}=i_{p k}(D)
$$

The per-unit power $p$ is a function of $m, d_{p}, d_{s}$ and $\delta$. Note that the value of $p$ can be both positive or negative. For dual active bridge operation with fixed $m$ and $p$, it is possible to determine a set of optimal modulation parameters $\left(d_{p}, d_{s}\right.$ and $\left.\delta\right)$ such that rms or peak of the inductor current is minimized [10], [12]. The RMS current minimization problem is mathematically stated below,

$$
\min _{\substack{0 \leq d_{p}, d_{s},|\delta| \leq 1, \mathrm{zVs} \\ p=p\left(m, d_{p}, d_{s}, \delta\right)}} i_{r m s}\left(m, d_{p}, d_{s}, \delta\right)
$$

For peak current minimization, the objective function is $i_{p k}\left(m, d_{p}, d_{s}, \delta\right)$. The optimal strategy for rms current minimization can be found in [10] whereas the strategy for peak
TABLE I: Boundary power levels [10]

\begin{tabular}{c|c|c}
\hline \hline & $p_{c 1}$ & $p_{c 2}$ \\
\hline$m \leq 1$ & $\frac{\pi m^{2}(1-m)}{2}$ & $\frac{\left(1-m^{2}\right) \pi}{2 m}\left(-1+\frac{1}{\sqrt{\left(1-m^{2}\right)}}\right)$ \\
\hline$m>1$ & $\frac{\pi(m-1)}{2 m}$ & $\frac{m \pi}{2}\left(1-m^{2}+m \sqrt{m^{2}-1}\right)$ \\
\hline
\end{tabular}

TABLE II: Optimal solution: $i_{r m s}$ minimization [10]

\begin{tabular}{c|c|c}
\hline \hline & $|p| \in\left[0, p_{c 1}\right)$ & $|p| \in\left[p_{c 1}, p_{c 2}\right)$ \\
\hline$m \leq 1$ & $d_{p}^{*}=\sqrt{\frac{2|p|}{(1-m) \pi}}$ & $\pi d_{p}^{*}\left(1-\left|\delta^{*}\right|\right)=\pi m\left(2 d_{p}^{*}-d_{p}^{* 2}\right)-2|p|$ \\
& $d_{p}^{*}=m d_{s}^{*}$ & $d_{s}^{*}=1$ \\
\hline$m>1$ & $\left|\delta^{*}\right|=(1-m) d_{s}^{*}$ & $\left|\delta^{*}\right|=1-\sqrt{2 d_{p}^{*}-d_{p}^{* 2}-\frac{4|p|}{m \pi}}$ \\
\hline & $d_{p}^{*}=m d_{s}^{*}$ & $d_{p}^{*}=1$ \\
& $d_{s}^{*}=\sqrt{\frac{2|p|}{\pi m(m-1)}}$ & $\pi d_{s}^{*}\left(1-\left|\delta^{*}\right|\right)=\frac{\pi}{m}\left(2 d_{s}^{*}-d_{s}^{* 2}\right)-\frac{2|p|}{m^{2}}$ \\
\hline
\end{tabular}

TABLE III: Optimal solution: $i_{p k}$ minimization [12]

\begin{tabular}{c|c|c}
\hline \hline & $|p| \in\left[0, p_{c 1}\right)$ & $|p| \in\left[p_{c 1}, \frac{m \pi}{4}\right]$ \\
\hline$m \leq 1$ & $d_{p}^{*}=\sqrt{\frac{2|p|}{(1-m) \pi}}$ & $d_{p}^{*}=1-\sqrt{\left(1-\frac{4|p|}{m \pi}\right) \frac{(1-m)^{2}}{(1-m)^{2}+m^{2}}}$ \\
& $d_{p}^{*} \leq m d_{s}^{*}$ & $d_{s}^{*}=1$ \\
\hline \multirow{3}{*}{$m>1$} & $\left|\delta^{*}\right|=(1-m) d_{s}^{*}$ & $\left|\delta^{*}\right|=1-\sqrt{2 d_{p}^{*}-d_{p}^{* 2}-\frac{4|p|}{m \pi}}$ \\
& $d_{p}^{*} \geq m d_{s}^{*}$ & $d_{p}^{*}=1$ \\
& $d_{s}^{*}=\sqrt{\frac{2|p|}{\pi m(m-1)}}$ & $d_{s}^{*}=1-\sqrt{\left(1-\frac{4|p|}{m \pi}\right) \frac{(m-1)^{2}}{(m-1)^{2}+1}}$ \\
\hline
\end{tabular}

current minimization can be found in [12]. The absolute perunit power $|p|$ must be less than $\frac{m \pi}{4}$ for solution the rms or peak current optimization problem to exist.

Solution of the rms current minimisation problem leads to three operating regions. For $|p| \in\left[0, p_{c 1}\right]$ (indicated in Table I), the converter operates with the modulation parameters listed in first column of Table II. For $|p| \in\left[p_{c 1}, p_{c 2}\right], d_{p}^{*}=1\left(d_{s}^{*}=1\right)$ for $m \geq 1(m \leq 1)$ and the other two optimum parameters are obtained by solving simultaneous equations indicated in the second column of Table II. For absolute value of operating power in the range $\left[p_{c 2}, \frac{m \pi}{4}\right]$, the converter operates with simple phase shift (SPS) strategy $\left(d_{p}^{*}=d_{s}^{*}=1\right)$ where $\left|\delta^{*}\right|=1-\sqrt{1-\frac{4 p}{m \pi}}$. The expressions of the optimal RMS currents as functions of optimal modulation parameters for different modes of operation are given in [27], which are also listed in Table IV for reference.

Solution of the peak current minimization problem leads to two operating regions. For $|p| \in\left[0, p_{c 1}\right]$, the optimal modulation parameters are listed in first column of Table III. For $|p| \in\left[p_{c 1}, \frac{m \pi}{4}\right]$, the optimal modulation parameters are indicated in the second column of Table III. The expressions of the optimal peak currents are given in second column of Table IV. The polarity of $\delta^{*}$ is same as polarity of $p$ for all the three operating regions of optimal rms current solution and the two operating regions of optimal peak current solution.

For DC-AC operation, $p(t)$ and $m(t)$ vary over line cycle as per equations (4) and (5). The rms and peak of the inductor current also vary over the line cycle. The rms current over line cycle, $\left\langle i_{r m s}\right\rangle_{T}$ can be computed from the following integral,

$$
\left\langle i_{r m s}\right\rangle_{T}^{2}=\frac{1}{T} \int_{0}^{T}\left\langle i_{r m s}\right\rangle_{T_{s}}^{2} d t
$$


TABLE IV: RMS and peak current expressions with optimal modulation strategy [27]

\begin{tabular}{|c|c|c|c|}
\hline & Peak current & RMS current for $|p| \leq p_{c 1}$ & RMS current for $|p| \geq p_{c 1}$ \\
\hline$m<1$ & $\frac{\pi}{2}\left(d_{p}-m d_{p}+m \delta\right)$ & $\begin{array}{c}\frac{\pi^{2}}{12}\left(d_{p}{ }^{3} m-2 d_{p}{ }^{3}+3 d_{p}{ }^{2}+3 d_{p} d_{s}{ }^{2} m\right. \\
\left.-6 d_{p} d_{s} m+3 d_{p} \delta^{2} m-2 d_{s}{ }^{3} m^{2}+3 d_{s}{ }^{2} m^{2}\right)\end{array}$ & $\begin{array}{l}\frac{\pi^{2}}{12}\left(-2 d_{p}{ }^{3}-3 d_{p}{ }^{2} \delta m+3 d_{p}{ }^{2} m+3 d_{p}{ }^{2}\right. \\
+6 d_{p} \delta m-6 d_{p} m-2 d_{s}{ }^{3} m^{2}-3 d_{s}{ }^{2} \delta m\end{array}$ \\
\hline$m \geq 1$ & $\frac{\pi}{2}\left(\delta-d_{s}+m d_{s}\right)$ & $\begin{array}{l}\frac{\pi^{2}}{12}\left(-2 d_{p}{ }^{3}+3 d_{p}{ }^{2} d_{s} m+3 d_{p}{ }^{2}-6 d_{p} d_{s} m\right. \\
\left.-2 d_{s}{ }^{3} m^{2}+d_{s}{ }^{3} m+3 d_{s}{ }^{2} m^{2}+3 d_{s} \delta^{2} m\right)\end{array}$ & $\begin{array}{c}+3 d_{s}^{2} m^{2}+3 d_{s}^{2} m+6 d_{s} \delta m-6 d_{s} m \\
\left.-\delta^{3} m+3 \delta^{2} m-6 \delta m+4 m\right)\end{array}$ \\
\hline
\end{tabular}

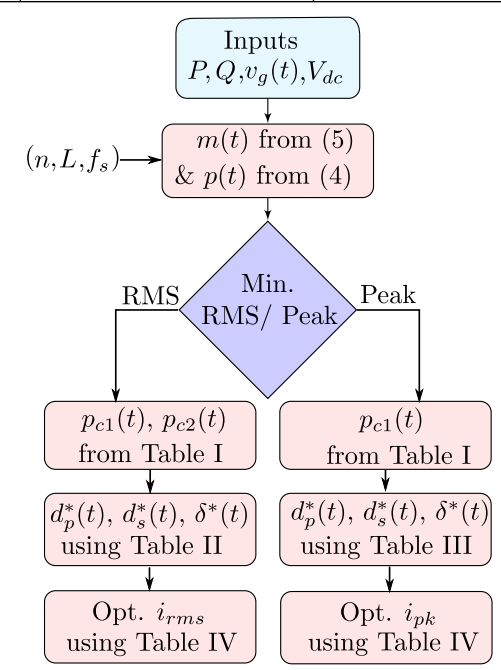

Fig. 4: Flowchart for determining $d_{p}^{*}(t), d_{s}^{*}(t), \delta^{*}(t)$ and the optimal $i_{r m s}$ and $i_{p k}$

The value of the line cycle rms current will be minimized if the rms current in each switching cycle is minimized. The peak current over a line cycle is the maximum current across all the switching cycles which can be minimized if $\left.i_{p k}\right|_{T_{s}}$ is minimized. For DC-AC operation with $f_{s}>f$, the values of $m$ and $p$ can be considered constant in a switching cycle. The optimal strategy for that given $m$ and $p$ which results in minimum rms/peak current can be derived using Table II/ III. This process is repeated over the entire line cycle to generate the optimal variation of the modulation parameters $d_{p}^{*}(t), d_{s}^{*}(t)$ and $\delta^{*}(t)$. Fig.4 shows the steps involved for determining the optimal modulation strategy for a given converter specifications and the values of $P$ and $Q$.

\section{UPF OPERATION: A SPECIAL CASE}

The strategy outlined in previous section can provide the optimal modulation strategy for bidirectional power flow at any arbitrary power factor. The case of unity power factor operation $(P>0$ or $P<0$ and $Q=0)$ is of utmost importance because most single-phase DC-AC power conversion systems are connected to grid and are required to draw/inject current at unity power factor. This section outlines the various cases that are encountered for UPF operation.

Depending upon the value of $M=\frac{n V_{p k}}{V_{d c}}$, two cases arise in AC operation-i) Case A- $M \leq 1$, the value of $m(t)=$ $M|\sin (\omega t)|$ is always less than 1 for the entire line-cycle and therefore, one needs to consider only first row of Table II for RMS current minimization or first row of Table III for peak current minimization; ii) Case B- if $M>1$, both $m(t) \leq 1$ and $m(t)>1$ occur for $0^{\circ} \leq \omega t \leq \gamma=\sin ^{-1}\left(\frac{1}{M}\right)$ and $\gamma \leq \omega t \leq 90^{\circ}$, respectively. This discussion is restricted for $0^{\circ} \leq \omega t \leq 90^{\circ}$ as the operation in $90^{\circ} \leq \omega t \leq 180^{\circ}$ will be mirror symmetric. For UPF operation $p(t)=\hat{p} \sin ^{2}(\omega t)$. Since the value of $m(t)$ varies over half of the line cycle, the power boundaries $p_{c 1}(t)$ and $p_{c 2}(t)$ (cf. Table I), used for optimal strategy determination, and the maximum attainable power, $\frac{\pi m(t)}{4}$, also vary. Based on the position of $p(t)$ with respect to these power boundaries, Case A and Case B have three and six sub-cases, respectively, which are discussed below.

Case A $(M \leq 1)$ : Fig. 5 a-c show the three sub-cases of Case A, where based on the magnitudes of $\hat{p}$ and $M$, the instantaneous power curve, $p(t)$, either doesn't intersect $p_{c 1}(t)$ and $p_{c 2}(t)$ boundaries (Case A-I), or only intersects $p_{c 1}(t)$ (Case A-II), or intersects both $p_{c 1}(t)$ and $p_{c 2}(t)$ boundaries (Case A-III). For example, let's consider Case A-III where $p(t)$ intersects both $p_{c 1}(t)$ and $p_{c 2}(t)$ boundaries, as shown in Fig. 5c. The intersecting angles, $\alpha_{1}$ and $\beta_{1}$, can be obtained by equating $p_{c i}(\omega t)=p(\omega t), i=1,2$. The expressions of $\alpha_{1}$ and $\beta_{1}$ are derived in Appendix. Based on the modulation strategy for optimal RMS inductor current, the positive half of the linecycle can be divided into three regions- i) S1 $\left(0 \leq \omega t \leq \alpha_{1}\right)$, where $0 \leq p(t) \leq p_{c 1}(t)$; ii) S2 $\left(\alpha_{1} \leq \omega t \leq \beta_{1}\right)$, where $p_{c 1}(t) \leq p(t) \leq p_{c 2}(t)$; iii) $\mathrm{S} 3\left(\beta_{1} \leq \omega t \leq \frac{\pi}{2}\right)$, where $p(t)>p_{c 2}(t)$. As $m(t) \leq 1$, the optimal modulation strategy is given by row-1 (R1) and column-1 (C1) of Table II for region $\mathrm{S} 1$. The corresponding optimal $i_{r m s}$ can be obtained by plugging $m(t)$ and the optimal modulation parameters $\left(d_{p}^{*}\right.$, $d_{s}^{*}$ and $\delta^{*}$ ) in the expression given in (R1,C2) (row-1 and column-2) of Table IV, which is applicable for $m(t) \leq 1$ and $0 \leq p(t) \leq p_{c 1}(t)$. Similarly, the optimal modulation strategies for regions S2 and S3 are (R1,C2) of Table II and SPS, respectively, and the corresponding $i_{r m s}$ expressions for both of these cases are given in C3 (column-3) of Table IV. Fig. 5f plots the modulation parameters corresponding to optimal $i_{r m s}$ of Fig. 5c. The optimal modulation parameters for the remaining two sub-cases of Case A are also shown in Fig. 5de. Similar exercise can be performed to find the modulation strategy and the expression corresponding to optimal $i_{p k}$.

Case B $(M>1)$ : In this case, one needs to consider five power boundaries, .viz, $\left.p_{c 1}\right|_{m \leq 1}(t),\left.p_{c 2}\right|_{m \leq 1}(t),\left.p_{c 1}\right|_{m>1}(t)$, $\left.p_{c 2}\right|_{m>1}(t)$ and the maximum power boundary. The intersecting angles of $\left.p_{c 1}\right|_{m \leq 1}(t)$ and $\left.p_{c 2}\right|_{m \leq 1}(t)$ with $p(t), \alpha_{1}$ and $\beta_{1}$, are same as Case A. It has been shown in Appendix that the intersection points between $p(t)$ and $\left.p_{c 1}\right|_{m>1}(t),\left.p_{c 2}\right|_{m>1}(t)$ in the range of $\left[\gamma, \frac{\pi}{2}\right]$ can be either zero or one or two, depending upon the values of $\hat{p}$ and $M$. In the detailed analysis given in Appendix, it is shown that six sub-cases of Case-B can occur for $M>1$ and $0 \leq \hat{p} \leq \frac{\pi M}{4}$. Fig. $5 \mathrm{~g}$-i and $5 \mathrm{~m}-\mathrm{o}$ show these six cases where $\alpha_{2}, \alpha_{3}$ are two intersection points between $p(t)$ and $\left.p_{c 1}\right|_{m>1}(t)$ and $\beta_{2}, \beta_{3}$ are two intersection points between $p(t)$ and $\left.p_{c 2}\right|_{m>1}(t)$. Let, $(r, s)$ is an ordered pair where $r$ and $s$ denote number of intersection points of $p(t)$ with $\left.p_{c 1}\right|_{m>1}(t)$ and $\left.p_{c 2}\right|_{m>1}(t)$, respectively. Then, $(r, s)$ of Case B-I to B-VI are- $(1,1),(0,1),(0,0),(2,1),(0,2)$, and $(2,2)$, respectively. For each of these cases, one can find 


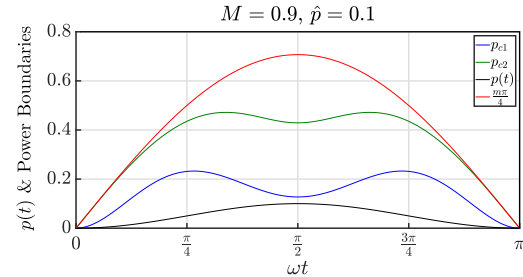

(a) Case A-I

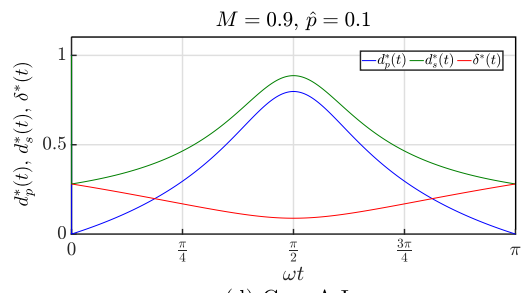

(d) Case A-I

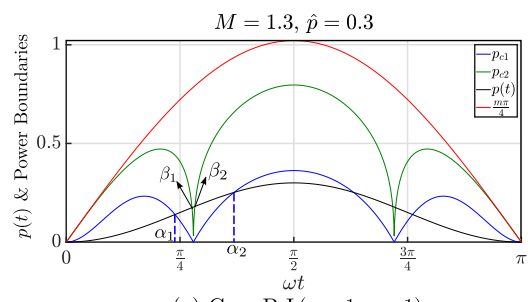

(g) Case B-I $(r=1, s=1)$

$M=1.3, \hat{p}=0.3$

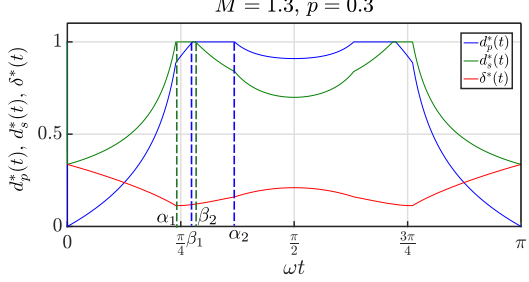

(j) Case B-I

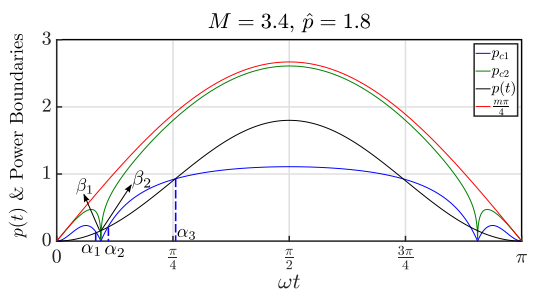

(m) Case B-IV $(r=2, s=1)$

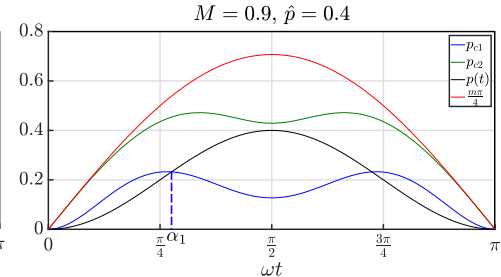

(b) Case A-II

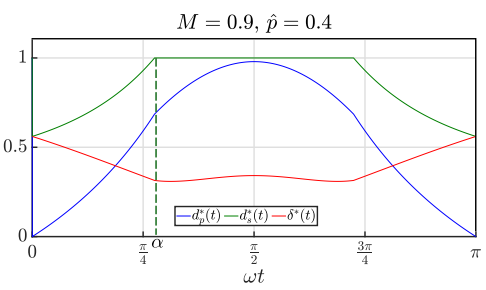

(e) Case A-II

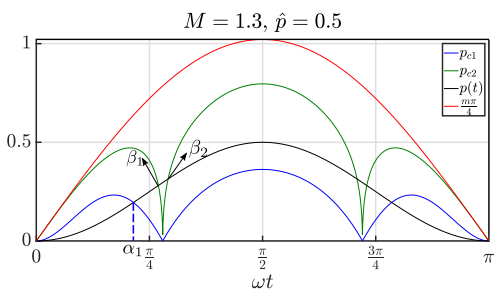

(h) Case B-II $(r=0, s=1)$

$M=1.3, \hat{p}=0.5$

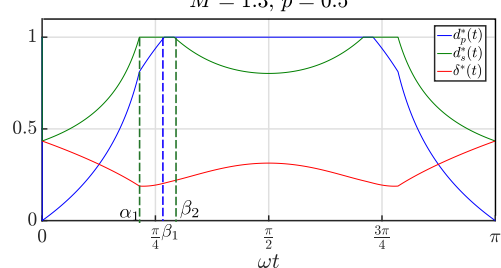

(k) Case B-II

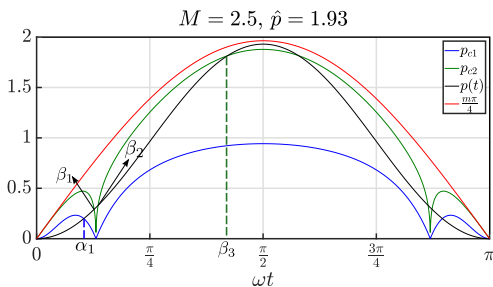

(n) Case B-V $(r=0, s=2)$

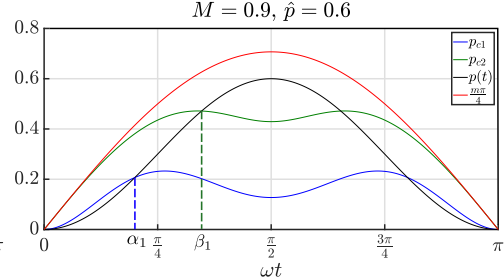

(c) Case A-III

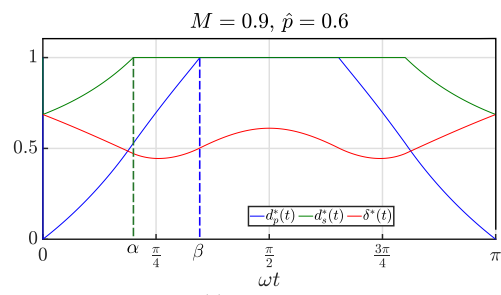

(f) Case A-III

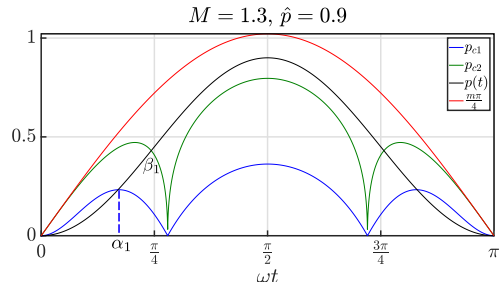

(i) Case B-III $(r=0, s=0)$

$M=1.3, \hat{p}=0.9$

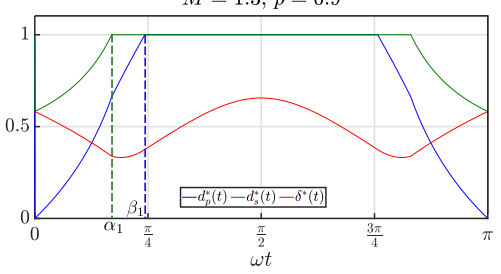

(1) Case B-III

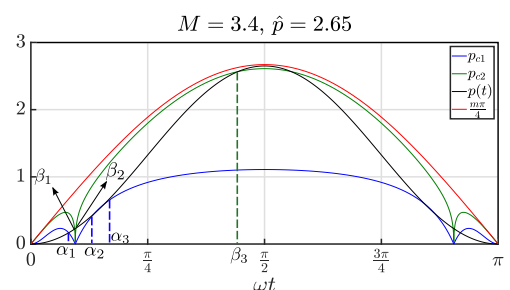

(o) Case B-VI $(r=2, s=2)$

Fig. 5: Power boundaries, $p(t)$, and the modulation parameters corresponding to optimal $i_{r m s}$ operation of nine possible cases of UPF operation.

the optimal strategies and the corresponding expressions in piecewise manner, as explained in the above example of Case A-III, using Table II, Table III, and Table IV. The optimal modulation parameters of Case B-I to B-III are shown in Fig. $5 \mathrm{j}-1$.

It is possible to determine the conditions on $\hat{p}$ for which each of the above nine cases arise. For example, Case A-III occurs when $p(t)>\left.p_{c 2}\right|_{m \leq 1}(t)$ or $\frac{p(t)}{\left.p_{c 2}\right|_{m \leq 1}(t)}>1$ anywhere in half of the line-cycle and $p(t)$ is less than or equal to maximum power boundary, i.e., $\frac{\pi m(t)}{4}$. Plugging $m=M \sin (\omega t)$ in the expression of $\left.p_{c 2}\right|_{m \leq 1}(t)$, as given in Table $\mathbf{I}$, and $p(t)=$ $\hat{p} \sin ^{2}(\omega t)$, the condition on $\hat{p}$ as function of $M$ (denoted by $k(M)$ ) can be derived so that the ratio, $\frac{p(t)}{\left.p_{c 2}\right|_{m \leq 1}(t)}$, is greater than 1 for any $\omega t \in\left[0, \frac{\pi}{2}\right]$. It can be shown that $\hat{p} \leq \frac{\pi M}{4}$ to satisfy $p(t) \leq \frac{\pi m(t)}{4}$ for $0^{\circ} \leq \omega t \leq 90^{\circ}$. Therefore, Case
A-III occurs when $k(M) \leq \hat{p} \leq \frac{\pi M}{4}$. Similarly, the condition, for which $p(t) \leq\left. p_{c 1}\right|_{m \leq 1}(t)$ for all $\omega t \in\left[0, \frac{\pi}{2}\right]$, is given by $0 \leq \hat{p} \leq l(M)$ (Case A-I). The detailed derivations of conditions $l(M), k(M)$ and other conditions for several cases of Case B are given in Appendix.

\section{EXPERIMENTAL AND Simulation Results}

For validating the modulation strategy, an experimental ACDC converter (Topology shown in Fig 1b) was designed with the specifications provided in Table V. Two separate design specifications are considered to test the converter operation, $M=0.51$ (Case A) and $M=1.3$ (Case B). The values of $n$ and $L$ are indicated for these two cases in Table V. In both of these cases, $P=1.2 \mathrm{~kW}, Q=0$ is maintained, which results in $\hat{p}$ to be equal to 0.39 and 0.52 for Case A and Case B, respectively. With these specifications, the proposed modulation strategy is validated through simulation in MATLAB-Simulink 
TABLE V: Hardware Specifications and $n$ and $L$ values for Case A and B

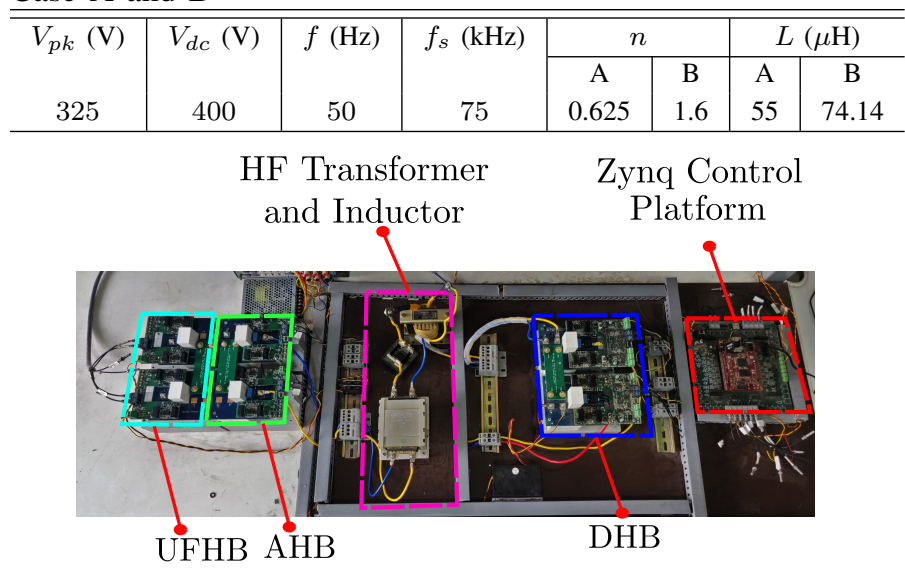

Fig. 6: Experimental Setup

with the ideal switches. As the converter operation for $M>1$ (Case B) consists of operation at both $m(t) \leq 1$ and $m(t)>1$, the experimental and simulated results are shown for $M>1$. In experimental set-up, $\mathrm{SiC}$ MOSFETS with voltage rating of $650 \mathrm{~V}$ (SCT3060AL) are used in the unfolder, AC side and DC side H-bridges. Zynq 7010 System on Chip (SoC) controller is used to generate the modulation signals. The experimental set-up is shown in Fig.6.

Fig. $7 \mathrm{a}$ and $7 \mathrm{~d}$ show the experimental and simulated sinusoidal phase voltage and current waveforms, respectively, at UPF operation with $M=1.3, \hat{p}=0.52$, which results in subcase B-II. The modulation strategy adopted here corresponds to optimal $i_{r m s}$. Following the algorithm of Fig. 4, the power boundaries $\left(p_{c 1}(t)\right.$ and $\left.p_{c 2}(t)\right), p(t)$, and the optimal modulation parameters for this operation are computed in real-time inside the controller. Fig. $7 \mathrm{c}$ and $7 \mathrm{i}$ show these signals over a line-cycle, which are taken as DAC (digitalanalog converter) outputs of the controller card. The simulated power boundaries are shown in Fig. 7f. It can be verified that these signals are identical to analytically obtained signals as shown in Fig.5h and 5k. The spectra of both experimental and simulated $i_{g}$ waveforms are indicated in Fig. 7b and 7e, where the fundamental RMS of the grid current is nearly 5.2 A. It can be seen that the current waveforms are free from low frequency harmonics. The THD (up to $50^{\text {th }}$ order harmonics) of the waveforms are close to $3 \%$ which is within the grid specifications (upto $5 \%$ ).

Fig. $7 \mathrm{~g}$ and $7 \mathrm{~h}$ show the experimental switching cycle waveforms of AHB and DHB converters during the peak and the zero crossing of $v_{g}(t)$, respectively, for optimal $i_{r m s}$ operation. Similar waveforms are obtained in simulation, which are shown in Fig. $7 \mathrm{j}$ and $7 \mathrm{k}$. At peak of $v_{g}(t)\left(\omega t=\frac{\pi}{2}\right)$, the DHB operates with $d_{p}=1$ (as $v_{a b}$ is square waveform), whereas $v_{c d}$ is a quasi-square waveform as the duty modulation occurs in AHB. Near zero crossing of $v_{g}(\omega t=0)$, both the AC side and DC side bridges operate with duty modulation. Therefore, operation in these two points conform with the optimal modulation strategy as indicated in $(\mathrm{R} 1, \mathrm{C} 1)$ (near zero crossing) and (R2,C2) (at peak) of Table II. Since power transfer is from DC to $\mathrm{AC}$ side for the entire line cycle during UPF operation, the voltage $v_{c d}$ lags $v_{a b}$ in both Fig. $7 \mathrm{~g}$ and
TABLE VI: Comparison of Theoretical, Simulation and experimental values at different operating conditions for $P=1.2$ $\mathrm{kW}, Q=0$

\begin{tabular}{c|c|ccc|ccc}
\hline \hline Case & Strategy & \multicolumn{3}{|c|}{$I_{r m s}(\mathrm{~A})$} & \multicolumn{3}{c}{$I_{p k}(\mathrm{~A})$} \\
& & $(\mathbf{T})$ & $(\mathbf{S})$ & $(\mathbf{E})$ & $(\mathbf{T})$ & $(\mathbf{S})$ & $(\mathbf{E})$ \\
\hline B & Opt. RMS & 4.22 & 4.25 & 4.8 & 10.3 & 10.6 & 11.4 \\
A & Opt. RMS & 9.52 & 9.66 & 9.61 & 22.28 & 22.8 & 23.4 \\
B & Opt. Pk. & 4.22 & 4.28 & 4.5 & 10.2 & 10.3 & 10.9 \\
\hline
\end{tabular}

7 h.

The converter performance is also verified for optimal peak strategy at UPF. The line cycle waveforms are identical to Fig.7a. Experimentally obtained optimal modulation parameters for this case is shown in Fig. 71, which is similar to Fig. 7i. Therefore, the switching cycle waveforms will be same as Fig. $7 \mathrm{~g}-7 \mathrm{~h}$ and Fig. $7 \mathrm{j}-7 \mathrm{k}$, and hence not shown here. This operation accords with the operation mentioned in $(\mathrm{R} 1, \mathrm{C} 1)$ (near zero crossing) and (R2,C2) (at peak) of Table III.

Table VI compares the theoretical $(\mathbf{T})$, simulation $(\mathbf{S})$ and experimental (E) values of inductor rms and peak currents for different operating cases and optimal strategies. A close agreement between the values can be observed for all the operating conditions. It can be also seen that for a given power transfer of $1.2 \mathrm{~kW}$ with Case A operation, the inductor rms and peak current magnitudes are significantly higher compared to Case B operation.

The switching transition at $t=t_{1}$ (of one of the DHB legs) in Fig.7g is considered for analysing the events occurring during soft switching. During this switching event, switch $S_{2}$ is turned off and $S_{1}$ is turned on after dead time (see Fig.8a). The inductor current $i_{1}$ is less than zero at this instant. Thus, the switch $S_{2}$ is conducting prior to its turn-off. The experimental waveforms of gate-pulses and the drain-source voltage of MOSFETS $S_{1}$ and $S_{2}$ participating in the transition are shown in Fig.8d. At $t=t_{A}$ in Fig.8d, the gate pulse of $S_{2}$ is turned-off. The gate voltage starts to reduce beyond this point and goes below threshold implying channel current is zero before $v_{C 2}$ starts to increase at $t=t_{B}$. This results in ZVS turn-off of $S_{2}$. The pole current now charges and discharges the capacitors $C_{2}$ and $C_{1}$ respectively (cf. Fig.8b and d). At $t=t_{C}$, the voltage $v_{C 1}$ reduces to zero and the body diode of $S_{1}$ starts to conduct (cf. Fig.8c). The turn-on of $S_{1}$ at $t=t_{D}$ happens when the voltage across it is zero (cf. Fig.8d). Similar observations were obtained for the remaining switching transitions of the converter.

\section{CONCLUSION}

This paper presents two optimal modulation strategies for a single-stage high-frequency link single-phase AC to DC converter that supports bi-directional power flow at any power factor. The $\mathrm{AC}$ is converted to a full-wave rectified $\mathrm{AC}$ through line frequency unfolding of an H-bridge converter incurring negligible switching loss. Two H-bridges with active devices (Dual Active Bridges) are connected through a high-frequency transformer and a series inductor to convert the full-wave rectified AC to a fixed DC. The two bridges are switched to generate two quasi-square waveforms with a phase shift. All three degrees of freedom in modulation, i.e., the duty cycles of the two quasi-square waveforms and the phase shift (TPS) between them, are used to devise two modulation methods. 


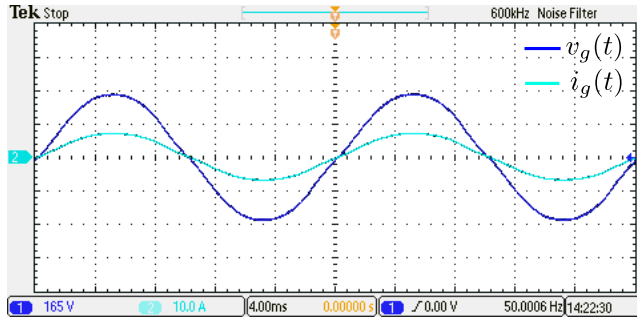

(a) Experimental $v_{g}(t)$ and $i_{g}(t)$

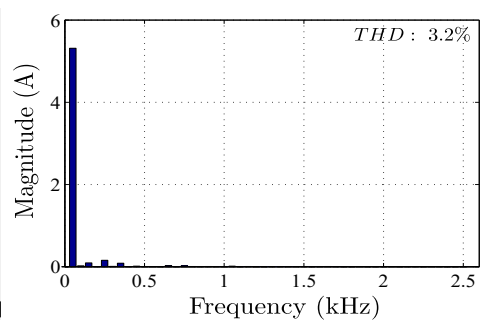

(b) Spectrum of experimental $i_{g}(t)$

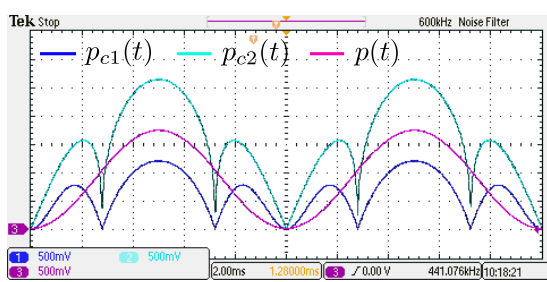

(c) Power Boundaries and $p(t)$ (experimental)

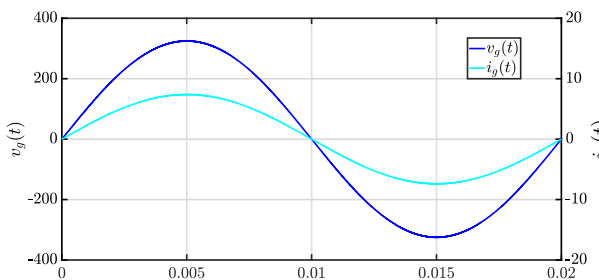

(d) Simulated $v_{g}(t)$ and $i_{g}(t)$

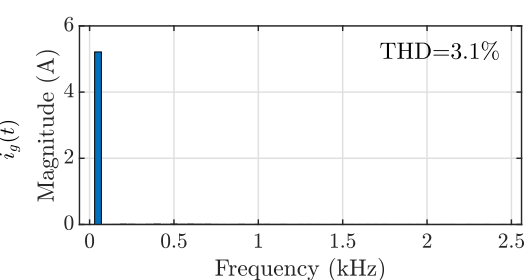

(e) Spectrum of simulated $i_{g}(t)$

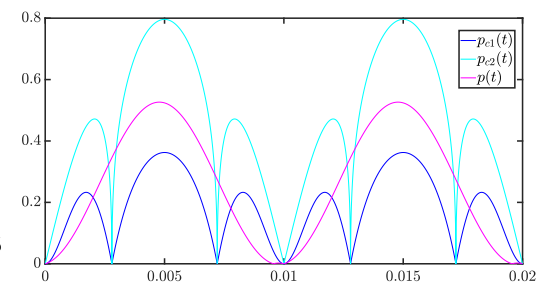

(f) Power Boundaries and $p(t)$ (simulated)

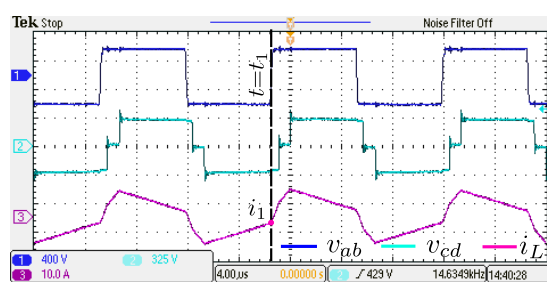

(g) $v_{a b}, v_{c d}$ and $i_{L}$ at peak of $v_{g}(t)$

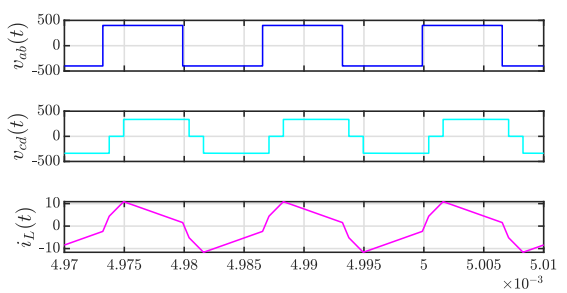

(j) Simulated $v_{a b}, v_{c d}$ and $i_{L}$ at peak of $v_{g}(t)$

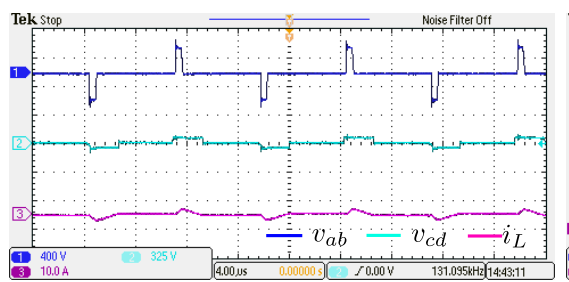

(h) $v_{a b}, v_{c d}$ and $i_{L}$ near zero crossing of $v_{g}(t)$

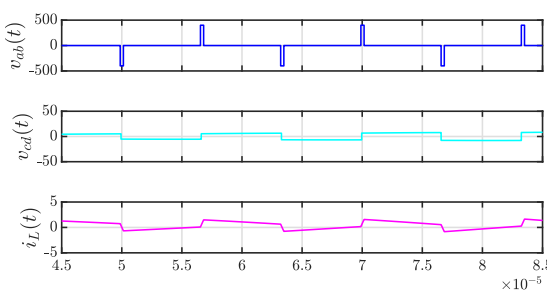

(k) Simu

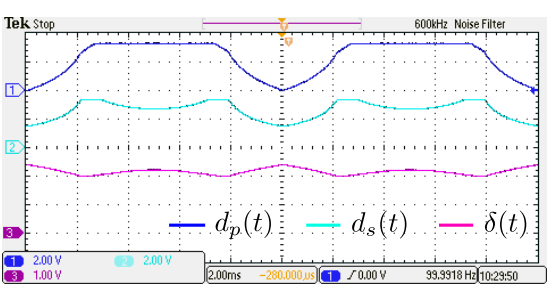

(i) Modulation parameters of optimal $i_{r m s}$

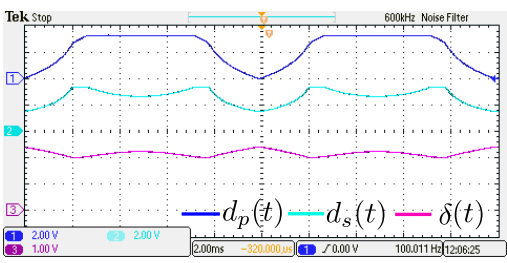

Fig. 7: Experimental and simulation results of the proposed optimal strategy at $M=1.3, \hat{p}=0.52$; a to k: optimal $i_{r m s}$ strategy; 1: optimal $i_{p k}$ strategy

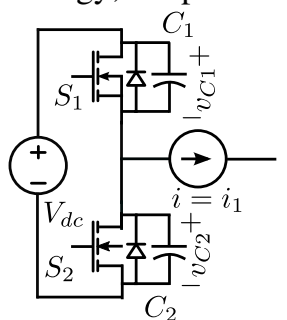

(a)

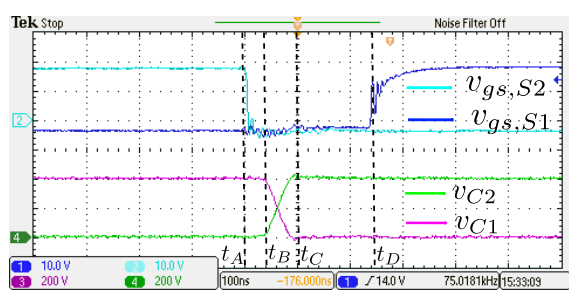

(b)

Fig. 8: (a) Equivalent circuit during switching event at $t=t_{1}$ in Fig.7g (b) Experimental waveform to validate ZVS

One minimizes the peak inductor current over the line cycle by minimizing the same over every switching cycle. The same is done for the minimization of the RMS inductor current for the other strategy. ZVS conditions are met for the entire line cycle, and no low-frequency harmonic is injected in the AC side line current. A step-by-step algorithm is given to find optimal TPS for specified average active and reactive power and sensed $\mathrm{AC}$ and DC port voltages. Steps are also provided for the theoretical computation of RMS and peak current stresses over a line cycle, which are essential for design. The unity

power factor (UPF) operation is discussed in detail, where it has been shown that nine cases may arise based on the ratio of the reflected peak of the AC port voltage and DC port voltage and the average active power. The proposed strategies are verified through simulation in MATLAB and experiments on a laboratory-scale hardware prototype at $230 \mathrm{~V}, 50 \mathrm{~Hz} \mathrm{AC}$ grid, $400 \mathrm{~V} \mathrm{DC}$, at UPF, and output power of $1.2 \mathrm{~kW}$.

\section{REFERENCES}

[1] M. Yilmaz and P. T. Krein, "Review of battery charger topologies, charging power levels, and infrastructure for plug-in electric and hybrid vehicles," IEEE Trans. on Power Electron., vol. 28, no. 5, pp. 21512169, 2013.

[2] M. Kasper, D. Bortis, G. Deboy, and J. W. Kolar, "Design of a highly efficient $(97.7 \%)$ and very compact $\left(2.2 \mathrm{kw} / \mathrm{dm}^{3}\right)$ isolated ac-dc telecom power supply module based on the multicell isop converter approach," IEEE Trans. on Power Electron., vol. 32, no. 10, pp. 7750$7769,2017$.

[3] C. A. Gallo, F. L. Tofoli, and J. A. C. Correa Pinto, "Two-stage isolated switch-mode power supply with high efficiency and high input power factor," IEEE Trans. on Ind. Electron., vol. 57, no. 11, pp. 3754-3766, 2010.

[4] M. Cacciato, A. Consoli, R. Attanasio, and F. Gennaro, "Soft-switching converter with hf transformer for grid-connected photovoltaic systems," IEEE Trans. on Ind. Electron., vol. 57, no. 5, pp. 1678-1686, 2010. 
[5] R. L. Steigerwald, "A comparison of half-bridge resonant converter topologies," IEEE Trans. on Power Electron., vol. 3, no. 2, pp. 174 $182,1988$.

[6] M. Kheraluwala, R. Gascoigne, D. Divan, and E. Baumann, "Performance characterization of a high-power dual active bridge dc-to-dc converter," IEEE Trans. Ind. Appl., vol. 28, no. 6, pp. 1294-1301, 1992.

[7] S. Li, J. Deng, and C. C. Mi, "Single-stage resonant battery charger with inherent power factor correction for electric vehicles," IEEE Trans. Veh. Technol., vol. 62, no. 9, pp. 4336-4344, Nov 2013.

[8] D. Patil and V. Agarwal, "Compact onboard single-phase EV battery charger with novel low-frequency ripple compensator and optimum filter design," IEEE Trans. Veh. Technol., vol. 65, no. 4, pp. 1948-1956, April 2016.

[9] A. Pal and K. Basu, "A partially soft-switched dc/ac high frequency link unidirectional converter for medium voltage grid integration," in 2015 National Power electronics Conference (NPEC), Dec 2015.

[10] A. Tong, L. Hang, G. li, X. jiang, and S. Gao, "Modeling and analysis of dual-active-bridge isolated bidirectional dc/dc converter to minimize rms current with whole operating range," IEEE Trans. Power Electron., vol. PP, no. 99, pp. 1-1, 2017.

[11] F. Krismer and J. Kolar, "Closed form solution for minimum conduction loss modulation of dab converters," IEEE Trans. Power Electron., vol. 27, no. 1, pp. 174-188, 2012.

[12] S. Shao, M. Jiang, W. Ye, Y. Li, J. Zhang, and K. Sheng, "Optimal phase shift control to minimize reactive power for a dual active bridge dc dc converter," IEEE Trans. on Power Electron., vol. 34, no. 10, pp. $10193-10205$, Oct 2019.

[13] K. Vangen, T. Melaa, and A. K. Adnanes, "Soft-switched highfrequency, high power DC/AC converter with IGBT," in PESC '92 Record. 23rd Annual IEEE Power Electronics Specialists Conference, 1992, pp. 26-33 vol.1.

[14] M. H. Kheraluwala and R. W. De Doncker, "Single phase unity power factor control for dual active bridge converter," in Conference Record of the 1993 IEEE Industry Applications Conference Twenty-Eighth IAS Annual Meeting, 1993, pp. 909-916 vol.2.

[15] N. D. Weise, G. Castelino, K. Basu, and N. Mohan, "A single-stage dual-active-bridge-based soft switched ac-dc converter with open-loop power factor correction and other advanced features," IEEE Trans. on Power Electron., vol. 29, no. 8, pp. 4007-4016, 2014.

[16] R. Baranwal, G. F. Castelino, K. Iyer, K. Basu, and N. Mohan, "A dualactive-bridge-based single-phase ac to dc power electronic transformer with advanced features," IEEE Trans. on Power Electron., vol. 33, no. 1 pp. 313-331, 2018.

[17] L. Zhu, A. R. Taylor, G. Liu, and K. Bai, "A multiple-phase-shift control for a sic-based ev charger to optimize the light-load efficiency, current stress, and power quality," IEEE Journal of Emerging and Selected Topics in Power Electronics, vol. 6, no. 4, pp. 2262-2272, 2018.

[18] A. K. Bhattacharjee and I. Batarseh, "Sinusoidally modulated ac-link microinverter based on dual-active-bridge topology," IEEE Trans. on Ind. Appl., vol. 56, no. 1, pp. 422-435, 2020.

[19] D. Sha, S. Wang, and J. Zhang, "A single stage natural power factor corrector based on dual active bridge dc-dc converter without inner current tracking loop," IEEE Trans. on Power Electron., pp. 1-1, 2020.

[20] J. Zhang, D. Sha, and P. Ma, "A dual active bridge dc-dc based single stage ac-dc converter with seamless mode transition and high power factor," IEEE Transactions on Industrial Electronics, 2021.

[21] J. Everts, F. Krismer, J. Van den Keybus, J. Driesen, and J. W. Kolar, "Optimal zvs modulation of single-phase single-stage bidirectional dab ac-dc converters," IEEE Trans. on Power Electron., vol. 29, no. 8, pp. 3954-3970, 2014.

[22] F. Jauch and J. Biela, "Single-phase single-stage bidirectional isolated zvs ac-dc converter with pfc," in 2012 15th International Power Electronics and Motion Control Conference (EPE/PEMC), 2012, pp. LS5d.1-1-LS5d.1-8.

[23] Y. Cho, W. Cha, J. Kwon, and B. Kwon, "High-efficiency bidirectional dab inverter using a novel hybrid modulation for stand-alone power generating system with low input voltage," IEEE Trans. on Power Electron., vol. 31, no. 6, pp. 4138-4147, 2016.

[24] J. Lu, G. Liu, H. Bai, A. Brown, P. M. Johnson, M. McAmmond, and A. R. Taylor, "Applying variable-switching-frequency variable-phaseshift control and e-mode gan HEMTs to an indirect matrix converterbased EV battery charger," IEEE Trans. Transport. Electrific., vol. 3, no. 3 , pp. 554-564, 2017.

[25] F. Jauch and J. Biela, "Combined phase-shift and frequency modulation of a dual-active-bridge AC-DC converter with PFC," IEEE Trans. on Power Electron., vol. 31, no. 12, pp. 8387-8397, 2016.

[26] D. Das and K. Basu, "Optimal design of a dual active bridge dc-dc converter," IEEE Trans. on Ind. Electron., pp. 1-1, 2020.

[27] D. Das and K. Basu, "Modulation strategy to minimise rms and peak currents in dual active bridge converter," in 2020 IEEE Energy Conversion Congress and Exposition (ECCE). IEEE, 2020, pp. 969974.

\section{APPENDIX}

\section{Intersecting angles of $p(t)$ with power boundaries}

The intersection angle of $p(t)$ with $\left.p_{c 1}\right|_{m \leq 1}(t)$ is given in (10). The intersection angle of $p(t)$ with $\left.p_{c 2}\right|_{m \leq 1}(t)$ is given by the solution of (11) in the range $[0,1]$, where $x=\sin \left(\beta_{1}\right)$. The two intersection angles of $p(t)$ and $\left.p_{c 1}\right|_{m>1}(t)$ are denoted by $\alpha_{2}$ and $\alpha_{3}$; and the two intersection angles of $p(t)$ and $\left.p_{c 2}\right|_{m>1}(t)$ are denoted by $\beta_{2}$ and $\beta_{3}$. These angles can be found by determining the solutions of $(12)$ and (13) in the range $\left[M^{-1}, 1\right]$. Here, $\sin \left(\alpha_{2}\right)=y=\sin \left(\alpha_{3}\right)$ and $\sin \left(\beta_{2}\right)=z=\sin \left(\beta_{3}\right)$.

$$
\begin{gathered}
\alpha_{1}=\sin ^{-1}\left(\frac{1}{M}-\frac{2 \hat{p}}{M^{3} \pi}\right) \\
y^{3}-\frac{\pi}{2 \hat{p}} y+\frac{\pi}{2 M \hat{p}}=0
\end{gathered}
$$

\section{Conditions on $\hat{p}$ for three sub-cases of Case A to occur}

$$
\begin{aligned}
& \frac{4 M \hat{p}}{\pi^{2}} x^{4}-\frac{4 M^{2} \hat{p}}{\pi} x^{3}+M^{3} x^{2}+\frac{4 \hat{p}}{\pi} x-M=0 \\
& \frac{4 M \hat{p}}{\pi} z^{3}-\frac{M^{4} \pi^{2}-4 \hat{p}^{2}}{M^{2} \pi^{2}} z^{2}-\frac{4 \hat{p}}{M \pi} z+1=0
\end{aligned}
$$

Consider the ratio $\frac{p(t)}{p_{c 1}(t)}$, as given in (14). The maximum value of this ratio occurs when $\omega t=\frac{\pi}{2}$. If the maximum value is ensured to be less than or equal to unity, $p(t) \leq p_{c 1}(t) \forall \omega t \in\left[0, \frac{\pi}{2}\right]$. This is ensured if $\hat{p} \leq \frac{\pi M^{2}(1-M)}{2}=l(M)$. The ratio $\frac{p(t)}{p_{c 2}(t)}$ can be similarly defined as in (15). It is possible to show that the maximum value of this ratio occurs at $\omega t=\frac{\pi}{2}$ and the maximum value is given in (16). If this maximum value is ensured to be less than or equal to unity, $p(t) \leq p_{c 2}(t) \forall \omega t \in\left[0, \frac{\pi}{2}\right]$. This is ensured if $\hat{p} \leq \frac{\pi\left(1-M^{2}\right)}{2 M}\left(-1+\frac{1}{\sqrt{1-M^{2}}}\right)=k(M)$.

$$
\frac{p(t)}{p_{c 1}(t)}=\frac{2 \hat{p}}{\pi M^{2}(1-M \sin (\omega t))} \quad \frac{p(t)}{p_{c 2}(t)}=\frac{2 M \hat{p} \sin ^{3}(\omega t)}{\pi\left[M^{2} \sin ^{2}(\omega t)-1+\sqrt{1-M^{2} \sin ^{2}(\omega t)}\right]} \quad \text { (15) }\left.\quad \frac{p(t)}{p_{c 2}(t)}\right|_{\max }=\frac{2 M \hat{p}}{\pi\left[M^{2}-1+\sqrt{1-M^{2}}\right]}
$$

\section{Conditions on $\hat{p}$ for six sub-cases of Case B to occur}

In this discussion, we want to find the conditions for which $\left.p_{c 1}\right|_{m>1}(t)$ and $\left.p_{c 2}\right|_{m>1}(t)$ intersect $p(t)$ or the polynomials of (12) and (13) have real roots in the interval $\left[\frac{1}{M}, 1\right]$. Note, (12) and (13) can be generalised as (17), where all the coefficients are positive. It can be shown that both of these polynomials have positive values at $\frac{1}{M}$, i.e., $f\left(\frac{1}{M}\right)>0$. Other two important properties of the function of (17) are: $f(-\infty)=-\infty, f(\infty)=\infty$. As $f\left(\frac{1}{M}\right)>0$ and $f(-\infty)=-\infty$, at least one real root exists in the range $\left(-\infty, \frac{1}{M}\right)$. As third order polynomial can have maximum three real roots, therefore, three cases can occur: i) one root, ii) two roots, or iii) no root exists in the range of $\left[\frac{1}{M}, 1\right]$.

$$
f(x)=a x^{3}-b x^{2}-c x+d ; a, b, c, d>0
$$

$$
f(1) \leq 0 \equiv(a+d) \leq(b+c)
$$




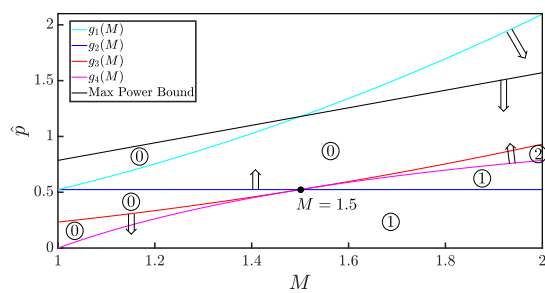

(a) Inequalities of (20), maximum power boundary(b) Inequalities of (21), maximum power bound-

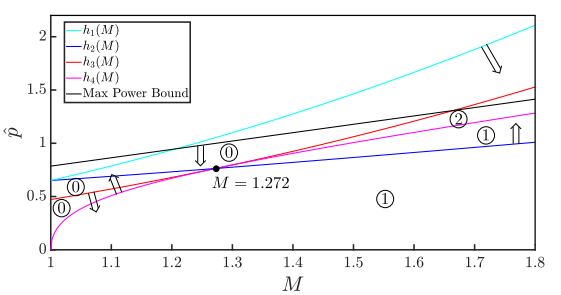

ary

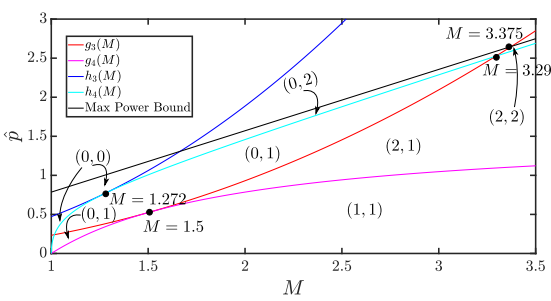

(c) Six possible sub-cases of Case B

Fig. 9

As $f\left(\frac{1}{M}\right)>0, f(1) \leq 0$, as given in (18), implies only one root exists in the range of $\left[\frac{1}{M}, 1\right]$. Four conditions, as given in (19), need to be satisfied simultaneously for the existence of two roots in $\left[\frac{1}{M}, 1\right]$. Here, $x_{\text {min }}$ is the local minima of $f(x)$. When none of these conditions are met, third case arises where there is no root in $\left[\frac{1}{M}, 1\right]$.

$$
\begin{aligned}
& x_{\text {min }}=\frac{b+\sqrt{b^{2}+3 a c}}{3 a} \geq \frac{1}{M} \\
& f\left(x_{\text {min }}\right) \leq 0 \Longrightarrow b^{2} c^{2}+4 a c^{3}+4 b^{3} d-27 a^{2} d^{2}+18 a b c d \geq 0 \quad \text { (19c) } \\
& \begin{array}{l}
x_{\text {min }}=\frac{b+\sqrt{b^{2}+3 a c}}{3 a} \leq 1 \\
f(1) \geq 0 \equiv(a+d) \geq(b+c)
\end{array}
\end{aligned}
$$

When $a, b, c$ and $d$, corresponding to (12), are substituted in (19), four conditions of (20) are obtained. Similarly, four conditions of (21) are obtained when the coefficients of (13) are substituted in (19). Fig. 9a shows the four constraints of (20) in $(M, \hat{p})$ plane, the arrow marks on these figures show the regions where the inequalities of (20) are satisfied. From our previous discussion, one can now easily find the number of intersecting points between $\left.p_{c 1}\right|_{m>1}(t)$ and $p(t)$ in $(M, \hat{p})$ and these numbers are marked within circles in Fig. 9a. Similarly, the number of intersection points between $\left.p_{c 2}\right|_{m>1}(t)$ and $p(t)$ in different regions of $(M, \hat{p})$ plane are marked in Fig. $9 \mathrm{~b}$.

$$
\begin{aligned}
\hat{p} \leq \frac{\pi M^{2}}{6}=g_{1}(M) \quad(20 \mathrm{a}) \quad \hat{p} & \geq \frac{\pi}{6}=g_{2}(M) \\
\hat{p} & \leq \frac{\pi(\sqrt{2}-1)}{2} M^{2}=h_{1}(M) \\
\hat{p} & \leq \frac{\pi}{\sqrt{22+10 \sqrt{5}}} M^{2}=h_{3}(M)
\end{aligned}
$$

$$
\begin{aligned}
& \hat{p} \leq \frac{2 \pi M^{2}}{27}=g_{3}(M) \quad(20 \mathrm{c}) \quad \hat{p} \geq \frac{\pi}{2}\left(1-\frac{1}{M}\right)=g_{4}(M) \\
& \text { a) } \quad \hat{p} \geq \frac{\pi M}{4}\left(\sqrt{9 M^{4}-2 M^{2}+1}-\left(3 M^{2}-1\right)\right)=h_{2}(M) \\
& \quad \hat{p} \geq \frac{\pi}{2} M \sqrt{M^{2}-1}\left(M-\sqrt{M^{2}-1}\right)=h_{4}(M)
\end{aligned}
$$

To determine all possible sub-cases of Case $\mathrm{B}$, one needs to consider all the above inequalities. It is possible to show that consideration of $g_{3}, g_{4}, h_{3}, h_{4}$ and maximum power boundary is sufficient to derive all possible cases in terms of number of intersection points of $p(t)$ with $\left.p_{c 1}\right|_{m>1}(t)$ and $\left.p_{c 2}\right|_{m>1}(t)$. Fig. 9c shows these boundaries for $1 \leq M \leq 3.5$. The number of intersection points of $p(t)$ with $\left.p_{c 1}\right|_{m>1}(t)(r)$ and $\left.p_{c 2}\right|_{m>1}(t)(s)$ are indicated by an ordered pair of the form $(r, s)$. One can see six possible cases are indicated in Fig. 9c- $(0,0),(0,1),(1,1),(0,2),(2,1)$ and $(2,2)$. Case $(2,2)$ appears after $g_{3}$ crosses $h_{4}$ at $M=3.29 . g_{3}$ also crosses the maximum power boundary at $M=3.375$. For $M>3.375, g_{4}<h_{4}<$ maximum power boundary; therefore, no new case arises after $M=3.375$. Note, $h_{4}$ asymptotically approaches $\frac{\pi M}{4}$, or the maximum power boundary when $M \rightarrow \infty$. From Fig. $9 \mathrm{c}$, one can identify the regions of the six sub-cases of Case $\mathrm{B}$ in $(M, \hat{p})$ plane. 